\title{
Quehaceres feministas, anudando y desanudando al Estado
}

\author{
Feminist tasks, knotting and unknotting the State
}

Claudia Anzorena*

Quehacer: ocupación que ha de hacerse

"Porque soy una poeta negra que hace su trabajo, vengo aquí a preguntarte: ¿Estás tú haciendo el tuyo?” (Audre Lorde)

\begin{abstract}
Resumen
¿Qué tenemos para decir las feministas sobre el Estado y las políticas públicas? El interrogante nos provoca tanto por las transformaciones que, en las últimas décadas, han experimentado los Estados en relación con las mujeres, como por los cambios en los discursos y prácticas políticas de los feminismos, y la articulación entre ambos procesos.

Después de más de 40 años de que "la condición de la mujer" ingresara en los espacios estatales latinoamericanos, los devenires de los procesos de institucionalización dan lugar a más preguntas que respuestas. Resulta necesario ir más allá del debate sobre la efectividad/inefectividad de las políticas y desplazar el eje hacia los quehaceres de los feminismos y cómo se van anudando con las formas y función del Estado, a través de la instalación de reivindicaciones que se consideran justas.
\end{abstract}

Palabras clave: Feminismos, Estado, Sentidos, Prácticas.

\footnotetext{
*Se graduó como licenciada en Sociología en 2002 (FCPyS, UNCuyo) en Mendoza, provincia en la que vive. Es especialista en género y políticas públicas (2004, PRIGEPP, FLACSO). En 2004, obtuvo una beca doctoral del Consejo Nacional de Ciencia y Técnica (CONICET- Argentina), gracias a la cual alcanzó los grados académicos de magíster en Política y Gestión de la Educación (2008, UNLuján) y doctora en Ciencias Sociales (2009, FCS, UBA). Desde 2000, ha participado en diferentes proyectos de investigación y ha publicado artículos en revistas científicas nacionales e internacionales, capítulos de libros y artículos de difusión. Es autora del libro "Mujeres en la trama del Estado. Una lectura feminista de las políticas públicas" (Mendoza, Ediunc, 2013). Como activista, integra la colectiva feminista La Malonacolectiva feminista (Mendoza) y la Campaña Nacional por el Derecho al Aborto Legal Seguro y Gratuito (Argentina).
} 


\begin{abstract}
What have feminists got to say about the State and public policies? This query is triggered both by the transformations undergone in the last few decades by the national states vis à vis women and by the changes in the feminisms' political discourses and practices, and the articulation between these two processes. More than 40 years have gone by since "the condition of women" entered Latin American state-run spaces: the evolution of institutionalization processes generates more questions than answers. It is therefore necessary to think beyond the debate around policy effectiveness/ineffectiveness, and to focus on feminisms' endeavors, analyzing how these endeavors tie with the State's forms and function through the installation of claims that are deemed rightful.
\end{abstract}

Keywords: Feminisms, State, Senses, Practices.

Fecha de recepción: Marzo 2019

Fecha de aprobación: Junio 2019

\title{
Prólogo
}

Resultó un placer para mi participar del Seminario Internacional "Actualidad política de los feminismos latinoamericanos", organizado por el Núcleo de Investigación en Género y Sociedad Julieta Kirkwood (FACSO-Universidad de Chile), realizado en agosto de 2018 en Santiago de Chile. El nudo que nos propusimos desenredar eran las formas emergentes e históricas en que los movimientos feministas latinoamericanos vienen planteando sus luchas y reivindicaciones para transformar el mundo. Tuvimos la oportunidad de dialogar con/entre feministas y feminismos de diferentes campos como la academia, las organizaciones o colectivas de base, las instituciones, desde diferentes puntos de posicionamientos: edades, antiespecistas, separatistas, disidentes sexuales, mapuches, radicales, institucionales, etc.

Fue un espacio enriquecedor del que traje una gran cantidad de interrogantes para seguir pensando. Además, me encontraba en un estado de embelesamiento porque a la semana siguiente del Seminario, el 8 de agosto, se trataría en el Senado Argentino la Ley de Interrupción Voluntaria del Embarazo que contaba con media sanción de la Cámara de Diputados/as.

Todos los momentos de condensación de las luchas y procesos históricos que venimos impulsando desde hace décadas, en consonancia o en tensión con nuestros feminismos prurales, nos exigen pensarnos en nuestras formas de vincularnos hacia adentro y hacia afuera de este movimiento heterogéneo, contradictorio, complejo que movemos con nuestras prácticas.

Hago esta breve prólogo antes de compartir lo que expuse en el seminario para recuperar el lugar desde el que hablo y nos hablamos. Necesitamos robustecer la legitimidad de 
nuestras discusiones, argumentos y posiciones, porque vivimos un momento de ofensiva feroz de los integrismos religiosos antiderechos más recalcitrantes que buscan deslegitmimarnos. Requerimosun posicionamiento epistemológico y político claro que muestre claramente cómo nuestras formas de construir conocimiento, si bien son situadas, se basan en la observación crítica y científica de los procesos sociales y no de especulaciones.

\section{Introducción}

Después de más de 40 años de que la denominada "condición de la mujer" (desfavorable claramente) ingresara en los espacios institucionales como un problema social del que los Estados debían encargarse, se han producido de manera extensa e intensiva investigaciones, balances, opiniones, críticas que versan sobre la eficiencia, efectos y devenires de estos procesos de institucionalización desde diferentes abordajes: el de mujeres en el desarrollo, la planificación de género, los derechos humanos, las alternativas del sur, los estudios poscoloniales, el ecofeminismo, la economía feminista, el feminismo popular, la crítica neoliberal, etc. (Aguinaga et al., 2012). Este repertorio da lugar a más preguntas que respuestas y, por tanto, a la necesidad de debatir sobre la relación entre Estados y feminismos; entre reivindicaciones feministas e intervención del Estado en pos de garantizar (algunos) derechos reconocidos ${ }^{1}$.

Mi intención es reflexionar teórica y políticamente en torno a la institucionalización que conseguimos como movimientos de mujeres y feministas, y dar algunos esbozos sobre cuáles son las paradojas y encerronas en que nos sitúa los anudamientos con el Estado. Se trata de exponer algunas inquietudes que me surgen de posicionarme en la confluencia entre mi trabajo (empleo) académico y mi activismo feminista, en ese espacio que habito, que integra academia y activismo. Si desde lo académico investigo sobre las políticas públicas que se dirigen directa o indirectamente a mujeres (en general definidas como madres, vulnerables y víctimas y escasamente como ciudadanas), desde el activismo me mueve la búsqueda de alternativas (políticas y prácticas) ante la insuficiencia y fragmentación de las medidas estatales.

Partimos de una perspectiva feminista, antirracista, anticapitalista, situada y crítica, que hace hincapié en las diferencias sexo-genéricas y las desigualdades de poder que de ellas se desprenden, como constitutivas de todas las relaciones sociales. Preguntarnos, desde esta perspectiva, por los anudamientos con el campo estatal es provocador tanto por las transformaciones que, en las últimas décadas, han experimentado los Estados con relación a la intervención en los asuntos considerados de la esfera doméstica, como por los cambios en los discursos y prácticas políticas de los feminismos, y la articulación entre ambos procesos.

La exposición gira en torno a dos cuestiones que he ido observando en varios contextos: una, que las relaciones entre Estado y feminismos son provisorias y contingentes; y otra, que estos anudamientos/desanudamientos nos ponen en una

\footnotetext{
${ }^{1}$ El tema que se desarrolla en este escrito continúa indagaciones de un artículo publicado en la RevlISE N¹1. Vol 11. abril - septiembre 2018 (Anzorena, 2018).
} 
paradoja, porque le exigimos respuestas a instituciones (generalmente al Estado) que se sostienen en la opresión, explotación y expropiación de los cuerpos (especialmente de los subalternizados, sexuados, racializados, empobrecidos).

\section{Desde dónde hablo}

Para escribir estas reflexiones me ubico en diferentes posiciones que explicitaré de modo de fundamentar que este discurso no es especulación u opinión, sino que es el producto de reflexiones políticas - proveniente de los feminismos - y teóricas -proveniente de las ciencias sociales, más específicamente de la sociología-.

Llevo 20 años analizando desde una lógica cualitativa cómo se piensan, se diseñan, implementan y afectan las políticas cuyas destinatarias o administradoras directas o indirectas - son mujeres. He analizado políticas de diferentes tipos: de género, de salud, socioeconómicas, laborales y en menor medida educativas (Anzorena, 2012; 2013; 2014). En el análisis buscamos considerar dos dimensiones para ir a la médula de las políticas entendidas como procesos organizacionales ${ }^{2}$, la dimensión histórica y la dimensión político-institucional. La primera, la dimensión histórica hace referencia a quién pone la cuestión en la escena política; cuáles son las fuerzas sociales que se aglutinan en una coalición capaz de sustentar el posicionamiento político de cierta demanda y requerir su instalación en la sociedad. La segunda refiere a la dinámica del desarrollo y las contradicciones y tendencias del desdoblamiento político-institucional, es decir, qué hace y cuánto tiempo lleva que una necesidad o una situación socialmente problemática se transforme en demanda política y pase a ser parte de la agenda de problemas socialmente reconocidos o vigentes, y por tanto atendidos por el Estado (Fleury, 1997).

En cuanto a mi posicionamiento político soy feminista, tengo privilegios que provienen de mi raza, de mi clase e inclusive de mi género, pero busco ponerlos en cuestión para que sean una herramienta de transformación. Como activista, desde 2000, he estado involucrada de manera colectiva en diferentes acciones para instalar en el espacio público sentidos feministas, para denunciar vulneración de derechos o situaciones injustas, para demandar la sanción o cumplimiento de leyes, para acompañar mujeres que quieren abortar, etc. Si bien mi recorrido personal es largo, y ha habido momentos de mayor y menor condensación, el año 2018 es señero, porque entre marzo y agosto se debatió en el Congreso de la Nación Argentina la legalización del aborto voluntario.

Estos meses de audiencias y debates implicaron que todos los poderes públicos y la sociedad escucharan, atendieran y tomaran posición en torno a nuestros argumentos sobre la reivindicación que disputa con mayor intensidad el control sobre los cuerpos

\footnotetext{
2 No entendemos las políticas y sus procesos de implementación como un conjunto de elementos orientados al logro de metas prefijadas, sino como procesos que implican redes de relaciones y tensiones que se dan entre la esfera técnica y la esfera política que desbordan ampliamente a las metas preestablecidas y donde el conflicto es propio de los procesos sociales. De este modo, las organizaciones no son entidades estructuradas y rígidas, ni los planes sociales la simple concreción de un proyecto o una idea planificada, sino que son el conjunto de negociaciones, tensiones y relaciones de fuerza que las constituyen (Beltrán Llavador, 2005). Se puede profundizar en esta postura en Anzorena, 2013.
} 
gestantes: el aborto. Las masivas manifestaciones en las calles y en las redes sociales, si bien no lograron la sanción de la ley, mostraron para el mundo la legitimidad que han adquirido algunas demandas feministas en el Cono Sur y la capacidad de movilización que hemos alcanzado desde los movimientos de mujeres, feministas y disidencias sexuales en el país, a través de nuestras prácticas políticas y de construcción de sentidos en torno a la justicia.

En la marea de estos movimientos, la Campaña Nacional por el Derecho al Aborto Legal, Seguro y Gratuito adquirió un protagonismo práctico y simbólico inusitado. Esta Campaña, que lanzamos el 28 de mayo de 2005 en diferentes puntos de Argentina (entre ellos Mendoza, donde vivo) mostró la potencia del capital social y político acumulado durante esos 13 años. A la vez que el pañuelo, el color verde que la caracterizó y el slogan que impulsó desde el inicio - "educación sexual para decidir, anticonceptivos para no abortar y aborto legal para no morir" - se consolidaron como símbolo de la posición favorable a la despenalización y legalización del aborto, fue capaz de poner en marcha un intenso trabajo: primero en el Congreso, para sumar votos a favor del proyecto de ley propio; segundo, en el espacio público para ampliar apoyos y movilizar en todo el país y, tercero, en los medios de comunicación masivos para instalar el debate en todas las casas y los espacios sociales. En definitiva, esta construcción no se generó de manera espontánea, sino que es el fruto del trabajo político y organizativo cotidiano y concreto de quienes le ponen/mos el cuerpo, la mente y el tiempo a esta Campaña (Anzorena y Zurbriggen 2013) ${ }^{3}$.

En este sentido, la posibilidad de este debate introduce variables novedosas en los estudios sobre las relaciones entre Estado y reivindicaciones/movimientos feministas. ¿Cuáles serán estas variables y su alcance sobre la relación que nos convoca? Creo que lo estamos viendo y los seguiremos haciendo en el proceso de balance de todo lo ocurrido en estos meses y en las formas en que encaremos la lucha en los tiempos que vienen.

\section{Cambiar el foco: de la intervención estatal a los quehaceres feministas}

"Existe una considerable diferencia entre no tener poder y tener un poco de poder, la diferencia entre estar fuera de los foros de decisión y ejecución o ser una parte de ellos. La gente con poco poder puede perder la mayoría de las batallas, sin embargo, participan al menos e imponen ciertas condiciones" (Hernes, 2003, p.21-22).

Las relaciones entre movimientos feministas y Estado han sido (y son) complejas y cambiantes. Las feministas no siempre pensaron al ámbito estatal como un espacio necesario para instalar sus reivindicaciones. A lo largo de su historia fueron cambiando

\footnotetext{
${ }^{3}$ En 2013 la Campaña publicó la compilación El aborto como derecho de las mujeres. Otra historia es posible (Buenos Aires: Herramienta, 2013), donde se recogen diferentes visiones de la Campaña.
} 
las formas de relacionarse con el Estado, desde entenderlo como un lugar neutral donde la transformación se daría por la sola inserción y participación de las mujeres, hasta concebirlo como la herramienta privilegiada de opresión del patriarcado y, por tanto, sentar posiciones antiestatistas o anarquistas (Archenti, 1994).

A partir de la reinstauración democrática en Argentina, en diciembre de 1983, y la consecuente revalorización de los derechos humanos, las feministas sacaron sus reivindicaciones de lo privado y las plantearon en el espacio público y social. Parte de esta politización las llevó a desarrollar acciones para que se sancionaran leyes e implementaran medidas estatales tendientes a resolver algunas de sus demandas, construidas en torno a necesidades, derechos humanos y justicia. Estos procesos y sus efectos, en su complejidad, ponen en cuestión las formas en que los gobiernos procesan institucionalmente las demandas feministas y al campo estatal como espacio donde instalar las demandas. Este cuestionamiento no se reduce a dar cuenta de los límites de la institucionalización y la planificación social, entendidos como el fracaso de las políticas para solucionar los problemas que se supone atienden. Se trata de plantear la necesidad de pensar y buscar alternativas al escenario hoy imperante.

Desde un punto de vista teórico-político feminista y sociológico, considero tanto un desafío como una necesidad desplazar el eje de la pregunta: no hacer tanto hincapié en las políticas y en la eficacia de la intervención, sino en cómo los quehaceres de los feminismos se van anudando con las formas y función del Estado, entendido éste como un espacio de disputas de sentidos, de poder, de relaciones de fuerzas, etc. Los quehaceres feministas tienen que ver tanto con los debates teóricos políticos que se dan en su interior como con la construcción e instalación de las demandas hacia el exterior y con quiénes debaten en la arena política. Se trata de introducir la cuestión de cómo construimos poder para incidir, como citamos en el epígrafe: no es lo mismo tener mucha, poca o nada de peso en las decisiones políticas, y esto para todas/os/xs las/os/xs suejtoas/os/xs subalternos es de radical importancia (Hernes, 2003). Este camino nos puede dar claves para abordar los laberintos del Estado y permitirnos pensar reclamos institucionalizables, cuando éstos son provechosos en beneficio de la vida cotidiana, y alternativas no institucionales, cuando nuestras prácticas e inquietudes no logran salir del territorio de la ilegalidad o de la ilegitimidad.

Los procesos históricos que llevaron a que los Estados intervengan en relación con la vida de las mujeres y específicamente a considerar sus condiciones de desigualdad como un problema social, fueron impulsados por las propias mujeres, feministas y sujetxs no masculinxs, de diferentes modos, en diferentes épocas. La instalación del tema en el espacio público poco tiene que ver con la iniciativa de algún/a dirigente político/a en particular, sino que, más bien, son los procesos históricos (en los que participan múltiples actores sociales con diferentes posiciones y relaciones de poder) que se posicionan de tal modo que las dirigencias los atienden, ya sea porque les trae beneficios políticos o bien porque ya no pueden evitarlos ${ }^{4}$.

${ }^{4}$ El 8 de marzo de 2018 un portal de noticias publicó un artículo de opinión donde decía "las dos principales iniciativas en materia de derechos políticos de las mujeres fueron iniciativas de varones" haciendo referencia a Perón con relación al sufragio femenino y a Menem con la ley de cupos (Claudia Peiró, Infobae, 8/3/2018). Esta opinión muestra una gran ignorancia histórica y teórica 
La idea de que "la mujer" debe ser dócil y nunca perder la compostura, y de que "el varón" y las instituciones patriarcales, tienen potestad sobre las decisiones y los cuerpos de las mujeres y la familia, ha sido y es, una concepción que cala profundo en el imaginario colectivo (público y privado), que, si bien permanece vigente, hay cuestionamientos y transformaciones que son consecuencia del accionar feminista.

Con nuestros quehaceres las feministas desplegamos lo que Fraser llama nuevas dimensiones de la justicia, que ponen en cuestión y buscan transformar, lo que "normalmente" se entiende que es justo, para quién es justo y cómo se determina este qué y quién (los sentidos); pero también cuestionan y transforman cómo se plantean y negocian las reivindicaciones por derechos en el espacio político (las prácticas)(Fraser 2008).

¿Qué quiere decir esto?

Quiere decir que desde los feminismos se construyen sentidos que instalan, por ejemplo, que no es justo que las decisiones que tomamos sobre nuestras sexualidades y capacidades reproductivas tengan que ser gestionadas en la clandestinidad, con todo lo que esto implica. A la vez que introducen prácticas y acciones que transgreden los mecanismos (psicológicos, sociales, económicos, políticos, ideológicos) establecidos como "normales", "aceptables", "lógicos" de cómo se entiende el mundo y por tanto de cómo se instalan en el espacio público las demandas o se denuncian las injusticias. Es así como estas prácticas son disruptivas y muchas veces rechazadas, porque sacan a "las mujeres" del lugar de la subalternidad, las presentan como insolentes, desacatadas y las posicionan como sujetas autónomas, con derecho y capacidades para tomar decisiones.

En definitiva, como señala Bareiro (2012), las feministas hemos transformado las competencias del Estado y de las sociedades en relación con los sujetos no masculinos. Esto ha sido posible porque venimos denunciando la discriminación y politizando los problemas y necesidades que se fugan de lo privado, con la construcción de nuevos sentidos y la participación política y social - cada vez más masiva y visible - en diferentes tipos de organizaciones (colectivas autónomas, movimientos sociales, ONGs o instituciones públicas o privadas) y con diversas acciones y manifestaciones.

\section{La construcción de sentidos y prácticas desde un lugar situado}

Las manifestaciones públicas es una de las estrategias que utiliza el movimiento feminista (como otros movimientos sociales) para instalar en el espacio público sus demandas. Estas demandas son de diferentes órdenes, en un abanico que va desde las que apuntan a la transformación de las relaciones sociales hasta expresiones

cuando no mala intención. Del mismo modo que no podemos decir que Macri inició el debate sobre el aborto cuando las feministas argentinas vienen desde la reinstauración democrática denunciando las situaciones que las mujeres deben pasar por la clandestinidad del aborto, a la vez que en 2005 se articularon en la Campaña Nacional por el Derecho al Aborto Legal, Seguro y Gratuito, que lleva 13 años instalando el debate en el espacio público y en el Congreso de la Nación. 
espontáneas o coyunturales de necesidades específicas, pedidos de garantía de derechos vulnerados, cumplimiento de leyes, reclamos por justicia o exigencias concretas.

Las feministas, en los espacios de activismo, llevan a cabo innumerables acciones que buscan sensibilizar, concientizar y transformar las condiciones de subordinación de las mujeres, niñas, personas trans, disidentes sexuales. Se organizan colectivamente, para abordar de manera autónoma diferentes problemas, es decir, sin vinculación con las instituciones públicas o con la menor vinculación posible. Por ejemplo, actividades de acompañamiento a mujeres en situación de violencia 0 de aborto seguro con medicamentos. También, buscan emprender en diversos ámbitos formas de trabajo cooperativo para el autosostenimiento, para la asistencia mutua en caso de alguna catástrofe o accidente, para promover una economía sustentable y solidaria, crear redes y medios para la comunicación no sexista, instancias de formación popular y no formal, articulación de campañas, una infinidad de prácticas y acciones. Muchas de estas acciones no tienen como objetivo la exposición pública sino más bien una transformación a nivel cotidiano de las relaciones sociales.

En cambio, cuando lo que se busca es poner sobre el tapete un reclamo político para que se constituya en problema social, toma la forma de la manifestación pública. Como señala Patrick Champagne, plantear públicamente los malestares modifica su estatus, de ser problemas personales o locales pasan a ser problemas sociales que deben ser resueltos políticamente; de ser responsabilidad individual a ser responsabilidad colectiva (Champagne, 2013). El activismo colectivo cotidiano, de base, del barrio, comunitario, en general, mantiene un perfil bajo. Es decir, no busca necesariamente como interlocutor a las instituciones para la implementación de una política. Sin embargo, es de allí de donde surge un gran caudal tanto de conocimientos sobre las condiciones de vida concretas de las mujeres, niñas/es/os, personas trans y disidentes sexuales como de diferentes acontecimientos, que permiten elaborar demandas, y plantearlas como problemas y necesidades que deben ser resueltas políticamente.

Estas demandas que resultan del quehacer cotidiano, en las sociedades capitalistas Nancy Fraser (1991) las denomina necesidades "escurridizas" o "fugitivas" porque surgidas en el ámbito privado, lo trascienden, se escapan, se fugan, y se instalan en el espacio social y público, como demandas públicas y políticas, donde ingresan muchas otras voces en las disputas por los sentidos, las interpretaciones y las formas de satisfacer estas demandas. El movimiento feminista, señala la autora instala sus necesidades a través de diferentes prácticas "como un medio para formular y debatir los reclamos políticos: es un estilo (una jerga) en el que el conflicto político es actuado y a través del cual las desigualdades se elaboran y cuestionan simbólicamente" (Fraser, 1991: 3-4).

Estas necesidades se van a exponer - se van a actuar - frente a la sociedad, los medios de comunicación, las instituciones públicas y privadas, al Estado e inclusive al mercado. Cada uno de estos actores sociales tiene diferentes posibilidades de recibir, entender y atender estas demandas. En esta disputa entran en tensión los sentidos feministas con los de los distintos actores sociales que emiten opiniones de índoles que pueden ser desde administrativas, hasta terapéuticas o de expertos/as o religiosas. Entre estos, a los movimientos no se les escapa que, en un sistema capitalista, el Estado se 
trata de la principal institución social capaz de desplegar los recursos humanos, organizacionales y tecnológicos necesarios para afrontar la mayoría de los desafíos que se presentan en las sociedades (Oszlak 2006).

Nancy Fraser señala que los movimientos sociales (en un sistema capitalista) buscan medidas estatales para satisfacer sus necesidades y que la política de las necesidades tiene tres momentos interrelacionados entre sí:

"El primero es la lucha por establecer o por negar el estatuto político de una necesidad dada, la lucha por validar la necesidad como un asunto de legítima preocupación política o por clasificarlo como un tema no político. La segunda es la lucha sobre la interpretación de la necesidad, la lucha por el poder de definirla y así determinar con qué satisfacerla. El tercer momento es la lucha por la satisfacción de la necesidad, la lucha por asegurar o impedir la disposición correspondiente” (Fraser, 1991: 8).

En cada uno de estos momentos, cada actor o movimiento involucrado construye un conjunto específico, cultural e histórico, de recursos disponibles para exponer sus demandas (o para oponerse) en un espacio social donde se encuentran en competencia una gran diversidad de formas de discursos y prácticas sobre las necesidades de las personas (Fraser, 1991) ${ }^{5}$. Este punto se pone en acto cada vez que nos manifestamos en post de algún derecho.

Las políticas públicas son un vínculo entre el Estado, la sociedad y el mercado. Este vínculo no es sexualmente neutro ni tampoco imparcial, tanto porque la construcción misma del Estado moderno, capitalista y colonial se basó en la expropiación y explotación de los cuerpos y el tiempo de las mujeres y de los pueblos indígenas (Federici, 2010; Yañez, 2015; Segato, 2015; Valobra, 2015), como porque su accionar implica sujetos sociales (la burocracia estatal, las distintas fracciones de la burguesía, los trabajadores/as, los movimientos sociales, instituciones religiosas, organismos internacionales, etc.) que detentan posiciones e intereses desiguales y diferentes en la arena política (Anzorena, 2013). El Estado es a la vez un espacio y un conjunto de procesos, que envuelve un juego contradictorio de posiciones, representadas por y en distintos órganos y sectores de la burocracia estatal. Las políticas públicas son el resultado de la configuración de las relaciones de fuerza existentes en cada momento, pero en definitiva son elaboradas, diseñadas y ejecutadas por quienes detentan el poder en el Estado. La burocracia estatal es al mismo tiempo arena de lucha política (donde alternan diferentes actores representando intereses privados); y actor social con iniciativas, interlocutora de otros/as actores, intérprete de un conjunto de directrices políticas (Oszlak, 2006; Fleury, 1997). Es decir que las articulaciones entre reivindicaciones feministas y políticas públicas son provisorias, en cuanto depende de la

\footnotetext{
${ }^{5}$ A fines de 2018 hubo una escalada de "escraches" que mujeres jóvenes y adolescente realizaron en redes sociales acusando a varones por abusos, violencias e inclusive violación. Esta forma de exposición pública suscitó un debate muy fuerte en torno a las formas y a los espacios para denunciar. Independiente de este debate, que no ha sido saldado, es entendible que para estas jóvenes y adolescentes este tipo de escrache fuera el recurso que tenían disponible para hacer visible las situaciones de violencias que experimentan, en un contexto donde las instituciones son expulsivas cuando no inaccesible o sordas a sus demandas.
} 
forma y función del Estado, del momento histórico, de las relaciones de fuerza, de los anudamientos con los diferentes espacios en disputa (Anzorena, 2014).

En síntesis: en las últimas décadas el campo estatal se presenta para los movimientos feministas latinoamericanos como un espacio donde anudar sus demandas. Por su parte, los Estados aparentan ser receptivos a ciertos problemas. Pero hay un umbral intraspasable entre las reivindicaciones de estos movimientos y los límites estatales a la garantía de los derechos de las mujeres y les disidentes sexuales, en vistas a que, por propia conformación capitalista, colonial y heteropatriarcal del Estado, sus respuestas nunca serán las adecuadas para quienes las demandan.

Esta complejidad se hace más evidente cuando ponemos en diálogo planteos que buscan alternativas no institucionales para mejorar las condiciones de vida (Segato, 2015) o ponen en duda teórica la legitimidad del Estado como interlocutor para instalar demandas (Valobra, 2015). Porque si bien podemos ser conscientes de que "las herramientas del amo nunca desmontan la casa del amo" (Lorde, 2003: 115), nos encontramos con el hecho de que una parte importante, al menos la más visible, de los movimientos feministas en Argentina dirigen sus pedidos concretos principalmente a las instituciones estatales, al punto de plantear en manifestaciones consignas donde se pone fuertemente el acento en que el Estado es responsable. Basta mirar declaraciones y consignas de \#NiUnaMenos en las marchas del del 3 de junio de 2015 a la actualidad (https://niunamenos.com.ar/), o de la Campaña Nacional por el Derecho al Aborto Legal, Seguro y Gratuito ante las muertes de mujeres y niñas después que el Senado rechazara la ley Interrupción Voluntaria del Embarazo (http://www.abortolegal.com.ar/).

¿Qué significa que las instituciones estatales son responsables? Cada definición que hagamos de esa "responsabilidad" plantea diferentes interpretaciones de las demandas que estamos planteando y, por tanto, de las formas de satisfacerlas.

Si el Estado es responsable porque se origina sobre la base de relaciones desiguales de clase, de raza y de género, entonces precisamos plantear una subversión radical del sistema que sostiene al Estado.

Si es responsable en cuanto se ha comprometido a implementar políticas para garantizar el ejercicio y acceso a los derechos humanos que reconoció a nivel internacional, precisamos una reforma de toda la legislación y habilitar mecanismos de acceso.

Si es responsable porque se trata de la institución pública que debe aliviar o contener los problemas que produce el propio sistema capitalista, cisheteropatriarcal y colonialista que sostiene, con mayor presupuesto y medidas de discriminación positiva podemos atender el problema.

Tanto la demanda como la estrategia dependerán de la definición e interpretación que hagamos de nuestros quehaceres, y de estas definiciones dependerán las formas de relacionarse con el campo estatal. Definiciones que son contingentes y provisorias en el marco de las disputas sobre los sentidos. 


\section{Consideraciones finales}

A lo largo de este artículo buscamos reflexionar sobre la relación entre quehaceres feministas y su articulación con la arena política, centradas en las formas cómo construimos y planteamos nuestras demandas como feministas y cuáles son las respuestas que recibimos. Las demandas del movimiento feminista se han multiplicado, las reivindicaciones van en diferentes sentidos y se plantean ante diferentes actores/as e instituciones, sin embargo, a quien mayormente se le exige intervención es al Estado. Un Estado que, si bien se ha transformado en los últimos 30 años de democracia, sigue siendo reticente a transformar su visión de "las mujeres" como víctimas, vulnerables, madres, domésticas.

La instalación de una demanda o necesidad en el espacio público implica entrar en disputa directa con actores sociales que tienen el poder de intervenir y opinar sobre las vidas y los cuerpos de las mujeres y les disidentes sexuales. Confrontaciones y disputas en torno a quién obtendrá qué y cómo, en las que el movimiento feminista evidentemente participa cuando plantea sus manifestaciones públicas y otorga responsabilidades.

Esto pone a las reivindicaciones feministas en una situación paradójica con relación al campo estatal: el Estado (se) sostiene (en) un sistema que se basa en la explotación y expropiación de algunos cuerpos, a la vez que se constituye en la institución que debería garantizar las acciones para modificar las relaciones de desigualdad que en definitiva promueve. Es decir, a la institución que se le exige/solicita respuesta es la misma que se acusa de producir y reproducir las desigualdades de género, de clase y de raza.

En este sentido es importante pensar cuáles son los límites y posibilidades de poner al campo estatal como interlocutor de las demandas que surgen de nuestras construcciones de sentidos y prácticas cotidianas, sin tener una confianza ciega en que va a modificar las relaciones desiguales, pero tampoco pensando que nuestro poder es tan escaso que no tenemos posibilidad de incidencia en las decisiones políticas o no podemos plantear formas alternativas de transformación y abordaje de nuestros problemas.

\section{Bibliografía}

Aguinaga, Margarita, Lang, Miriam, Mokrani, Dunia, \&Santillana, Alejandra (2012): "Pensar desde el feminismo: Críticas y alternativas al desarrollo". En Más allá del 
Desarrollo. Grupo Permanente de Trabajo sobre Alternativas al Desarrollo, de Miriam Lang y Dunia Mokrani (comp.), 323. Buenos Aires: Fundación Rosa Luxemburg.

Anzorena, Claudia (2018): "El campo estatal en cuestión: brechas entre las demandas feministas y políticas públicas”, enRevIISE, Vol. 11, No 11, p. 213-227. Disponible en: http://www.ojs.unsj.edu.ar/index.php/reviise/article/view/226/pdf

Anzorena, Claudia (2014): "Aportes conceptuales y prácticos de los feminismos para el estudio del Estado y las políticas públicas". En Revista de Trabajo Social PLAZA PÚBLICA 7, no 11,p. 17-41.

Anzorena, Claudia (2013): Mujeres en la trama del Estado: una lectura feminista de las políticas públicas. Mendoza: EDIUNC.

Anzorena, Claudia (2012): "Planificación, educación y mujeres: miradas alternativas ¿Cómo leen los/as hacedores/as de las políticas sociales las experiencias de las mujeres?". En Revista Venezolana de Estudios de la Mujer. Dossier Las mujeres en las organizaciones: inclusión, gerencia y cambio (CEM Universidad Central de Venezuela) 17, no 38, p. 225-246.

Anzorena, Claudia \& Zurbriggen Ruth (2013) "Trazos de una experiencia de articulación federal y plural por la autonomía de las mujeres: La Campaña Nacional por el Derecho al Aborto Legal, Seguro y Gratuito en Argentina", en Zurbriggen y Anzorena (comp):El aborto como derecho de las mujeres. Otra historia es posible.Buenos Aires: Herramienta.

Archenti, Nélida (1994):“Las mujeres, la política y el poder. De la lógica del príncipe a la lógica de la acción colectiva” en D. Maffía y C. Kuschnir, Clara (Comps.): Capacitación política para mujeres: género y cambio social en la Argentina actual. Buenos Aires: Feminaria.

Bareiro, Line (2012): “Avances y desafíos para la participación política de las mujeres”, en Gherardi, Natalia(dir.) Lidera: participación en democracia. Experiencias de mujeres en el ámbito social y político en la Argentina, 27-36. Buenos Aires: ELA Equipo Latinoamericano de Justitica y Género.

Beltrán LLavador, Francisco (2005): Travesías de las organizaciones educativas (y otros desórdenes). Valencia: Germania.

Champagne, Patrick (2013): "La visión del Estado", en Bourdieu,Pierre (dir.): La miseria del mundo, 187-194. Buenos Aires: Fondo de Cultura Económica

Federici, Silvia (2010): Calibán y la bruja. Mujeres, cuerpo y acumulación originaria. Madrid: Traficantes de sueños. 
Revista Punto Género № 11. Junio de 2019

ISSN 0719-0417 / 5 - 17

Fleury, Sonia (1997): Estado sin ciudadanos. Seguridad social en América Latina. Buenos Aires: LUGAR.

Fraser, Nancy (2008): Escala de justicia. Barcelona: Herder.

Fraser, Nancy (1991): "La lucha por las necesidades: Esbozo de una teoría crítica socialista-feminista de la cultura política del capitalismo tardío". En: Debate feminista 3, p. 3-40.

Hernes, Helga (2003): El poder de las mujeres y el Estado del bienestar. Madrid: Vindicación feminista.

Lorde, Audre (2003): La hermana, la extranjera. Madrid: horas y Horas..

Oszlak, Oscar (2006): "Burocracia estatal: política y políticas públicas", en POSTData Revista de Reflexión y Análisis Político, n 11, p.11-56.

Segato, Rita (2015): "La norma y el sexo: frente estatal, patriarcado, desposesión, colonialidad", en: Marisa Belausteguigoitia Rius y Saldaña-Portillo, María Josefina (comp.) Des/posesión: género, territorio y luchas por la autodeterminación, p.125-164. México: Universidad Nacional Autónoma de México.

Valobra, Adriana (2015): "El Estado y las mujeres, concepciones en clave feminista." En Estudios Sociales del Estado 1, no 2, p. 32-57.

Yañez, Sabrina (2015): "De cómo las instituciones de salud pública regulan las experiencias de embarazo, parto y puerperio... y de lo que resta" (Mendoza, 2001 2013)". Tesis (Doctoral en Antropología). Buenos Aires: UBA., Facultad de Filosofía y Letras 